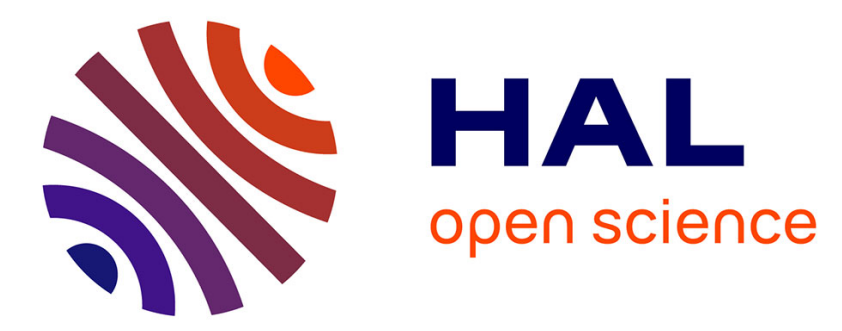

\title{
Surdité et mouvements anormaux péri-oraux chez une femme de 46 ans
}

\author{
Y. Péréon, G. Ahle, Y. Nadjar
}

\section{To cite this version:}

Y. Péréon, G. Ahle, Y. Nadjar. Surdité et mouvements anormaux péri-oraux chez une femme de 46 ans. Pratique Neurologique - FMC, 2019, 10, pp.147 - 151. 10.1016/j.praneu.2019.02.005 . hal-03486491

\section{HAL Id: hal-03486491 https://hal.science/hal-03486491}

Submitted on 20 Dec 2021

HAL is a multi-disciplinary open access archive for the deposit and dissemination of scientific research documents, whether they are published or not. The documents may come from teaching and research institutions in France or abroad, or from public or private research centers.
L'archive ouverte pluridisciplinaire HAL, est destinée au dépôt et à la diffusion de documents scientifiques de niveau recherche, publiés ou non, émanant des établissements d'enseignement et de recherche français ou étrangers, des laboratoires publics ou privés.

\section{다)(1) $(5$}

Distributed under a Creative Commons Attribution - NonCommerciall 4.0 International 
Surdité et mouvements anormaux péri-oraux chez une femme de 46 ans ${ }^{1}$. Deafness and abnormal perioral movements in a 46-year-old woman.

Yann PEREON (1)*, Guido AHLE (2), Yann NADJAR (3)

${ }^{1}$ Laboratoire d'Explorations Fonctionnelles, Centre de Référence Maladies Neuromusculaires Rares Atlantique-Occitanie-Caraïbes - FILNEMUS, Hôtel-Dieu, 1 Place Alexis-Ricordeau 44093 Nantes France

${ }^{2}$ Département de Neurologie, Hôpitaux Civils de Colmar, 68000 COLMAR

${ }^{3}$ Département de Neurologie, CRML (Centre de Référence des Maladies Lysosomales), UF Neuro-Génétique et Métabolisme, Hôpital Pitié-Salpêtrière, Boulevard de I'hôpital, 75013

PARIS

*Correspondance à :

Yann Péréon, Laboratoire d'Explorations Fonctionnelles, Centre de Référence Maladies Neuromusculaires Rares Atlantique-Occitanie-Caraïbes - FILNEMUS, Hôtel-Dieu, 1 Place Alexis-Ricordeau 44093 Nantes France

Yann.Pereon@univ-nantes.fr

\footnotetext{
${ }^{1}$ Cette confrontation a été présentée à la session de la Société Française de Neurologie aux JNLF 2018, tenues à Bordeaux. Elle a été sélectionnée, organisée puis mise en page par le Dr François SELLAL (Colmar).
} 


\section{Présentation de l'observation clinique (Guido Ahle, Colmar).}

Madame N., âgée de 46 ans, d'origine marocaine, était suivie en ORL pour un implant cochléaire. L'orthophoniste constata une baisse récente des capacités de la patiente à lire sur les lèvres, raison pour laquelle un bilan neurologique fut demandé.

A la reprise de son histoire on relevait une baisse de l'acuité auditive dès l'âge de 8 ans, d'évolution très lentement progressive, puis l'apparition d'une chute des paupières et de discrets mouvements anormaux péri-oraux, dont la patiente ne se plaignait pas.

II n'y avait pas de notion de troubles de la déglutition, pas d'intolérance à l'effort, pas de faiblesse segmentaire, pas de gêne respiratoire ou cardiaque. II n'y avait pas de notion de crise d'épilepsie.

Dans les autres antécédents, on notait que la patiente était née après un accouchement difficile, à domicile, avec probable souffrance néonatale. II n'y avait cependant pas de données anamnestiques suggérant un retard des acquisitions psychomotrices. II n'y avait pas de consanguinité ni de cas similaire dans la famille.

A l'examen clinique, on observait un ptosis bilatéral, plus marqué à gauche, une hypoacousie de perception bilatérale, des mouvements anormaux péri-oraux et une atrophie linguale avec fibrillations (vidéos 1 et 2). Les réflexes ostéo-tendineux étaient vifs et symétriques, mais sans être diffusés ni polycinétiques. Les réflexes cutanés plantaires se faisaient en flexion. Il n'y avait pas de déficit moteur, pas de troubles du tonus, de la sensibilité, pas d'ataxie ni de troubles de la coordination.

Le bilan biologique revint sans anomalie particulière pour la numération formule sanguine, l'ionogramme, la fonction rénale, la glycémie, le bilan lipidique. II n'y avait pas de syndrome inflammatoire biologique. Le bilan immunologique montrait une immuno-électrophorèse et des protéines sériques normales, une négativité des recherches du facteur rhumatoïde, des anticorps anti-nucléaires et des anticorps anti-muscle strié.

La ponction lombaire revint également sans anomalie particulière (absence de cellule, glycoet lactatorachie, protéinorachie et bilan immunitaire normaux). Une étude de I'ADN mitochondrial dans le sang ne montra pas de mutation MERFF et MELAS.

Une IRM cérébrale ne put être réalisée en raison de la présence des implants cochléaires. Un scanner cérébral était dépourvu d'anomalie.

Des potentiels évoqués visuels étaient normaux, comme un EEG.

L'EMG des quatre membres donnait les résultats suivants : 
- Conduction motrice: amplitudes évoquées musculaires distales normales aux 4 membres.

- Stimulation répétitive à $3 \mathrm{~Hz}$ : absence de décrément significatif sur les nerfs cubitaux droit et gauche, spinal gauche et facial gauche.

- Etude du nerf facial gauche avec blink reflex : normal à gauche, VII normal.

- Conduction sensitive : amplitudes normales aux membres supérieurs et inférieurs. Absence de ralentissement des vitesses de conduction sensitive.

- Détection : absence d'activité spontanée anormale. A l'effort, tracés neurogènes très chroniques avec potentiels géants au niveau de la langue et de la face mais normaux ailleurs ( $V$ moteur, sterno-cléido-mastoïdien, membres).

Le bilan cardiologique (ECG, échocardiographie transthoracique) et des explorations fonctionnelles respiratoires ne montrèrent pas d'anomalie. Quelle aurait été votre hypothèse diagnostique et quels examens complémentaires proposeriez-vous ?

\section{Discussion (Yann Péréon, Nantes)}

Le tableau clinique tel qu'il est présenté recèle plusieurs éléments caractéristiques qui méritent d'être individualisés par leur caractère inhabituel : la surdité, précoce (survenue à l'âge de 8 ans) et sévère (puisqu'ayant nécessité la pose d'un implant cochléaire) ; le ptosis bilatéral asymétrique, élément souvent en faveur d'une pathologie neuromusculaire; l'amyotrophie linguale, à importante valeur localisatrice; enfin, les mouvements involontaires péribuccaux, qui peuvent orienter dans beaucoup de directions mais prendre leur sens une fois confrontés aux autres symptômes affectant cette patiente.

Une première analyse qu'on effectuerait selon une approche standardisée de la neurologie serait de commencer par être uniciste sur un versant topographique. Où se trouve la lésion, est-elle centrale ou périphérique ? Si elle est centrale, cérébrale sus- ou sous-tentorielle, ou spinale etc ? Ce mode de raisonnement - d'abord 'où', puis 'comment' avant d'aborder le 'pourquoi' - est privilégié lorsqu'on débute en neurologie, mais il ne permet pas ici d'apporter la solution : les 4 éléments retenus renvoient certes vers le tronc cérébral (III, VII, VIII et XII èmes paires de nerfs crâniens), mais il nous est mentionné la normalité du scanner cérébral (qui n'est pas le meilleur outil d'imagerie du tronc mais a été effectué dans le contexte de l'implant cochléaire contre-indiquant la réalisation d'une IRM cérébrale), ainsi que du liquide céphalo-rachidien, dont on peut raisonnablement penser qu'il aurait été 
affecté par une pathologie locale du tronc suffisamment sévère pour atteindre plusieurs nerfs crâniens comme c'est le cas ici.

Prenons maintenant chacun de ces 4 éléments caractéristiques, en les mêlant les uns aux autres au gré du raisonnement clinique.

\section{Raisonner à partir du ptosis.}

Le ptosis, motif relativement fréquent de consultation, peut avoir de multiples origines (vasculaires, compressives...) et si l'on considère le ptosis chronique, renvoie notamment vers un éventail de pathologies musculaires. On oubliera le ptosis aponévrotique ou encore congénital. II est ici décrit comme bilatéral, asymétrique, mais non fluctuant, n'est pas associé à une diplopie, ou à un syndrome de Claude Bernard-Horner. L'hypothèse de la jonction neuromusculaire a pu paraitre séduisante, ce d'autant que les myasthénies à antiMusk peuvent s'accompagner d'un aspect particulier de la langue (avec une atrophie tripliquée) ; elle a été testée, mais la stimulation nerveuse répétitive est restée négative. La sensibilité de cet examen lors des myasthénies oculaires n'est pas optimale et il aurait pu être utile de lui adjoindre un EMG de fibre unique pour s'assurer de la normalité du jitter. L'absence d'antécédents familiaux ou de consanguinité, de double réponse en neurographie, sont d'autres éléments qui ne sont pas en faveur d'une myasthénie congénitale. De plus, I'EMG réalisé à la face a montré des tracés neurogènes chroniques avec unités motrices de grande taille. Cet aspect va à l'encontre de celui attendu dans la myasthénie ou dans les formes de myasthénies congénitales à connotation myopathique (ex: DoK7), ce d'autant plus que l'aspect habituel de l'EMG de la face est normalement pseudo-myogène. Des tracés 'pseudo-neurogènes' peuvent être rencontrés au cours d'authentiques myopathies, mais ils ne prennent jamais le caractère d'unités motrices géantes, encore moins au niveau de la face.

L'hypothèse d'une pathologie musculaire propre doit faire évoquer 2 causes classiques, finalement fréquemment rencontrées même si on reste dans le champ des maladies rares: le ptosis est quasi-constant dans la dystrophie myotonique de Steinert, il peut y être associé à une surdité, mais manquent ici les éléments cliniques ou paracliniques habituels (pas de myotonie clinique ou électrique, de cataracte, d'hypersomnie, de morphotype spécifique...). La dystrophie musculaire oculopharyngée n'est pas non plus suspectée, malgré l'atteinte linguale associée, dans le contexte d'un EMG en détection 
franchement neurogène. D’autres myopathies notamment congénitales ne seront pas non discutées ici.

L'association ptosis-surdité évoque immédiatement l'hypothèse mitochondriale. Si l'on peut facilement éliminer les classiques tableaux de MELAS et Kearns-Sayre (absence des autres atteintes habituelles telles qu'ophtalmoplégie externe, AVC, diabète, endocrinopathies, atteinte rénale, cardiopathie...) ou MERFF (les mouvements péri-buccaux auraient pu être des myoclonies), ce d'autant que l'observation mentionne la négativité de la recherche de mutations MELAS ou MERFF, il faut mentionner l'existence de syndromes rares comme le CODAS (Cerebral Ocular Dental Auricular Skeletal syndrome (LONP1), avec certes surdité, hémi-atrophie linguale, ptosis, mais aussi malformations osseuses, dentaires, cataracte congénitale, retard psychomoteur non observés ici). De nombreuses autres mitochondriopathies (ex: COXPD34, Wolfram syndrome, CAGSSS...) sont responsables de surdité précoce, mais l'absence d'association à d'autres stigmates cliniques permet de les oublier ici.

\section{$\underline{\text { Raisonner à partir des mouvements anormaux péribuccaux. }}$}

Les mouvements péribuccaux tels que présentés sur la vidéo de la patiente semblent de faible amplitude, erratiques, bilatéraux, impliquant plusieurs éléments musculaires de façon asynchrone. Ils n'apparaissent pas gênants, puisque la patiente ne s'en plaint pas. II ne semble pas s'agir de dyskinésies buccofaciales ou de myoclonies mais il pourrait plutôt s'agir de myokimies ou de fasciculations abondantes : un examen discriminant mais non réalisé chez cette patiente est un EMG de surface, qui permet de facilement différencier ces patterns, par la présence de PUM individuels ou groupés (fasciculations, doublets, multiplets), ou au contraire d'activité contractile à contenus beaucoup plus riches (dyskinésies ou même myoclonies). A eux seuls, ces mouvements péribuccaux ne permettent pas en tout état de cause de faire avancer le diagnostic.

\section{Raisonner à partie de l'atrophie linguale.}

L'atrophie linguale peut représenter l'élément déterminant, ce d'autant que l'examen en détection à l'aiguille a mis en évidence une dénervation motrice chronique : l'atteinte des 
deux XII entre ici dans un contexte d'atrophie neurogène bulbaire, avec un ensemble de causes souvent génétiques que l'on va faire défiler [1].

Pourrait-il s'agir d'une sclérose latérale amyotrophique? Non, même si les signes bulbaires sont présents au moment du diagnostic chez 30\% des patients SLA et si les formes à début bulbaire ne sont pas rares, y compris parmi les formes génétiques (expansions C9orf72 plus fréquemment que mutations sur SOD1). On peut souligner ici l'absence de signes pyramidaux, d'atteinte musculaire extra-faciale, que ce soit cliniquement ou à I'ENMG. Le ptosis n'est pas non plus un élément évocateur, tout comme la surdité.

L'amyotrophie bulbo-spinale de Kennedy peut être mentionnée, même si concernant très principalement des hommes compte tenu de la transmission récessive liée à I'X. Des formes frustes tardives ont été décrites chez des femmes hétérozygotes ou des doubles homozygotes [2]. L'absence de dénervation plus diffuse que bulbaire, d'anomalies des potentiels sensitifs à l'ENMG, de tremblement noté, l'âge et le sexe, la présence du ptosis, de la surdité (si l'on est uniciste) permettent d'éliminer ce diagnostic.

Amyotrophie linguale et fasciculations peuvent se rencontrer au cours du syndrome FOSMN de description relativement récente (facial onset sensory motor neuronopathy) [1]: survenant au cours des $5^{\text {ème }}-7^{\text {ème }}$ décennies, il est responsable d'une atteinte motrice mais aussi sensitive (paresthésies), affectant initialement la face, avec extension sur un mode rostro-caudal vers les épaules, les membres supérieurs puis inférieures. Outre l'amyotrophie linguale et les fasciculations, il peut comporter dysgueusie, dysphagie, dysarthrie, enrouement, amyotrophie massétérine et temporale, avec diminution des réflexes cornéen et nauséeux. L'ENMG met en évidence, outre la dénervation bulbaire, des anomalies du blink reflex (réponses R1 et R2 absents ou retardées) et une neuropathie axonale sensitivomotrice. Ces éléments manquent dans le cas présent et cette hypothèse peut être raisonnablement écartée.

Une autre cause d'atrophie bulbaire, qui plus est associée à une surdité, fait revenir aux mitochondriopathies: des mutations sur le gène $\mathrm{CHCHD10}$ qui encode une protéine mitochondriale en hélice, auraient pu être recherchées, mais le mode de transmission (autosomique dominant), l'existence de signes cérébelleux et pyramidaux, ici non notés, ne sont pas en faveur [3].

La forme dite de Madras de maladie motoneuronale, liée à une mutation A8302G dans I'ARNt mitochondrial se traduit par un tableau combinant surdité neurosensorielle, diplégie 
faciale, dysphagie, dysarthrie, enrouement, amyotrophie linguale, fasciculations [4]. Elle peut également comprendre une atteinte motrice prédominant aux membres supérieurs, des signes pyramidaux, une atrophie optique. L'ENMG montre une dénervation chronique active dans les territoires bulbaire et facial, mais aussi dans les membres, elle peut débuter de la $1^{\text {ère }}$ à la $5^{\text {ème }}$ décennie et son évolution est lente. Ne serait l'origine géographique de la patiente - du Maroc et non du sud de l'Inde, où cette pathologie est primitivement rapportée - cette étiologie aurait représenté une hypothèse plausible pour expliquer l'ensemble du tableau décrit ici.

Certaines neuropathies périphériques peuvent avoir un tropisme particulier pour la face: parmi les nombreuses formes de neuropathies de Charcot-Marie-Tooth, les formes CMT4C (gène SH3TC2) et CMT4D (gène NDRG1) incluent toutes deux une amyotrophie linguale et une surdité, mais l'atteinte (sensitivo)-motrice distale, le pied creux, l'évolution sévère, les anomalies importantes à l'ENMG ne sont pas observés dans le cas présent.

Une autre neuropathie pose plus question et pourrait expliquer le tableau actuel : les neuropathies amyloïdes avec mutation sur le gène codant la gelsoline peuvent être responsables d'une atteinte motrice restreinte à la face avec ptosis, amyotrophie linguale, hypotonie et relâchement cutané de la face, dysarthrie, dysphagie, mais aussi myokimies. II n'y a pas de déficit des membres, peu ou pas de troubles sensitifs (distaux) [5]. L'ENMG de la face objective les myokymies et des tracés neurogènes, comme ici. L'élément distinct est la surdité que présente la patiente, mais dont on peut toujours douter du lien avec le reste des signes rapportés.

Le syndrome de Brown-Vialetto-Van Laere est peut-être la solution au tableau clinique de la patiente: tous les éléments observés chez elle sont effectivement présents : ce syndrome associe dès l'enfance surdité de perception, atteinte bulbaire avec atrophie linguale, fasciculations, dysphagie et ptosis. Une caractéristique non retrouvée chez la patiente présentée est la fréquence de l'atteinte des muscles du cou, pouvant affecter la tenue de la tête et faire de la 'tête penchée' un signe d'appel chez l'adolescent. Il est lié à des mutations sur les gènes SLC52A3, SLC52A2 codant pour des protéines transmembranaires transporteurs de la riboflavine (vitamine B2), avec une transmission autosomique récessive et une présentation souvent sporadique. L'intérêt de cette hypothèse est la possibilité d'introduire un traitement par riboflavine permettant d'espérer améliorer ou au moins stabiliser le patient [6]. 


\section{Synthèse de la discussion.}

Au total, très peu d'hypothèses semblent devoir être retenues, qui pourraient expliquer l'ensemble du tableau présenté chez cette patiente : le syndrome de Brown-Vialetto-Van Laere paraît être l'étiologie la plus évidente; à défaut, une neuropathie amyloïde par mutation sur le gène GSN (gelsoline) est à rechercher. Enfin, en cas de négativité des deux précédentes, une cause mitochondriale (mutations sur le gène $\mathrm{CHCHD10}$ ou la forme de Madras de maladie du motoneurone) resterait à considérer.

De fait, le diagnostic repose sur des examens principalement génétiques avec analyse des gènes responsables de Brown-Vialetto-Van Laere (qui serait mon premier choix diagnostique), de la neuropathie amyloïde à gelsoline, éventuellement une analyse mitochondriale plus complète et une recherche de Kennedy par principe. Dans l'attente de ces examens, la présence d'anomalies du profil des acylcarnitines pourrait représenter un indice en faveur du Brown-Vialetto-Van Laere. La réalisation d'un bilan ophtalmologique complet, y compris électrophysiologique (ERG) voire d'une biopsie musculaire peut se discuter dans un second temps.

\section{Solution (Yann Nadjar, Paris)}

Effectivement devant ce tableau d'atteinte du motoneurone périphérique associé à une surdité, le syndrome de Brown-Vialetto-Van Laere (BVVL) est la meilleure hypothèse diagnostique.

Chez notre patiente la riboflavine plasmatique fut dosée et se révéla limite à 176 $\mathrm{nmol} / \mathrm{I}(\mathrm{N}=174-471)$. Le rapport pyruvate/lactate et le profil des acylcarnitines était normal. En revanche, l'analyse génétique de SLC52A 1,2 et 3 objectiva une mutation hétérozygote SLC52A2:c824G>T et une mutation hétérozygote SLC52A3: c.634C>T.

Le syndrome de BVVL a été décrit il y a plusieurs décennies comme une maladie de type dégénératif du motoneurone périphérique, de début pédiatrique, parfois familiale avec une transmission de type récessif, et associé à une atteinte auditive [7]. Le phénotype moteur est caractérisé par une atteinte fréquente des motoneurones bulbaires (dysarthrie, trouble de la déglutition, amyotrophie et fasciculations de langue) et pontiques (atteinte faciale inférieure mais aussi supérieure). Les muscles respiratoires sont également souvent atteints, mettant en jeu le pronostic vital. Enfin, les membres peuvent aussi être atteints, de 
même que les nerfs crâniens oculomoteurs. Des cas débutant chez le jeune adulte ont également été décrits.

Chez la patiente décrite, l'atteinte du motoneurone périphérique très chronique, et de localisation bulbaire (amyotrophie et fasciculations de langue) est compatible avec ce diagnostic. L'argument majeur en sa faveur est la présence d'une surdité, qui est la manifestation la plus fréquente du syndrome de BVVL. Dans cette maladie, il s'agit d'une neuropathie auditive, mise en évidence par des potentiels évoqués auditifs altérés alors que les oto-émissions acoustiques sont normales, témoignant d'un fonctionnement normal de la cochlée.

Récemment, deux gènes codant pour des transporteurs de la riboflavine ont été rapportés comme étant impliqués chez certains patients BVVL (SLC52A2 et SLC52A3) $[8,9]$. L'intérêt majeur de cette découverte est que ces patients avec déficit de transporteur en riboflavine (DTR) ont pu bénéficier d'une supplémentation orale de riboflavine à forte dose qui a modifié profondément l'histoire naturelle de leur maladie. Des patients sévèrement atteints ont pu être sevrés de la ventilation mécanique et récupérer une marche autonome [10]. Ces deux transporteurs ont une répartition tissulaire différente et permettent à la fois le transport du secteur digestif vers le secteur plasmatique et du secteur plasmatique vers le secteur tissulaire, notamment le système nerveux central [11] (figure 1).

La riboflavine est une vitamine hydrosoluble qu'on trouve dans beaucoup d'aliments et qui est utilisée dans l'organisme comme co-facteur de nombreuses réactions enzymatiques d'oxydo-réduction, via ses dérivés FAD et FADH. Parmi ces protéines nécessitant de la riboflavine (environ une soixantaine) figurent de nombreuses enzymes et transporteurs impliqués dans la beta oxydation mitochondriale des acides gras. Le déficit multiple en acyl-coA-deshydrogénases (MADD, Multiple Acyl CoA Dehydrogenase Deficiciency) est une maladie génétique de manifestation principalement musculaire liée à un déficit de transporteurs d'électrons (ETF et ETFDH) nécessitant le FAD, qui aboutit à une perturbation de la beta oxydation mitochondriale [12]. Le MADD se dépiste sur des anomalies spécifiques visibles sur le profil plasmatique des acylcarnitines, qui ont également été trouvées chez des patients avec mutations SLC52A2 ou SLC52A3, du fait d'un déficit fonctionnel en ETF et ETFDH lié à l'absence de FAD.

Chez la patiente décrite, le profil des acylcarnitines était normal, de même que le dosage sanguin de riboflavine, mais cela n'élimine pas le diagnostic de déficit de 
transporteur en riboflavine, car la sensibilité de ce signe n'est pas de $100 \%$, et cela semble d'autant plus vrai dans les formes modérées de la maladie à début tardif comme chez notre patiente. La génétique est donc indispensable pour confirmer le diagnostic: c'est ainsi qu'ont été trouvées chez elle deux mutations hétérozygotes de type substitution jamais rapportés auparavant et suspectes de pathogénicité, l'une dans le gène SLC52A2, l'autre dans le gène SLC52A3. Son tableau clinique est plus en faveur d'un défaut du gène SLC52A3, du fait de l'absence d'atrophie optique et surtout de neuronopathie sensitive qui sont fréquemment associés à la maladie du motoneurone dans les mutations SLC52A2. Cependant le DTR a toujours jusqu'à présent été rapporté comme une maladie récessive, ce qui est compatible avec l'histoire de la patiente, dont les parents ne sont pas atteints, l'affirmation du diagnostic nécessitant donc l'identification de deux mutations sur un même gène. La revue de la littérature montre cependant que chez des cas avérés de DTR avec tableau clinique évocateur et réponse au traitement par riboflavine à forte dose, une seule une mutation a pu être trouvée [11]. Pour les patients SLC52A3 à début tardif (au-delà de 10 ans), c'est en fait le cas pour la moitié des patients (7/13, revue de la littérature et cohorte française). Il est également possible que deux mutations sur deux transporteurs différents, comme ceci a été trouvé chez la patiente, puissent être responsable d'un DTR, mais cela n'a jamais été rapporté. Le diagnostic de DTR est donc très probable chez elle. Cependant certains éléments pourraient contribuer à le confirmer définitivement : i) la caractérisation de la surdité en faveur d'une neuropathie auditive, ii) la détection d'une deuxième mutation a priori complexe (intronique ? autre ?) dans SLC52A3 (voire SLC52A2), et enfin iii) une réponse clinique ou électrophysiologique à une supplémentation par riboflavine à forte dose.

\section{Déclaration d'intérêts}

Les auteurs n'ont pas transmis leurs liens d'intérêts éventuels en relation avec cet article. 


\section{Références.}

1. Manole A., Fratta P., Houlden H. Recent advances in bulbar syndromes. Current Opinion in Neurology $2014 ; 27(5)$ : 506-514.

2. Mariotti C, Castellotti B, Pareyson D, et al. Phenotypic manifestations associated with CAG-repeat expansion in the androgen receptor gene in male patients and heterozygous females: a clinical and molecular study of 30 families. Neuromuscul Disord $2000 ; 10: 391-7$.

3. Nguyen, H. P., Van Broeckhoven, C., van der Zee, J. ALS Genes in the Genomic Era and their Implications for FTD. Trends in Genetics 2018 ; 34(6) : 404-423.

4. Govindaraj P, Nalini A, Krishna N, Sharath A, Khan NA, Tamang R, Gourie-Devi M, Brown RH, Thangaraj K. Mitochondrial DNA variations in Madras motor neuron disease. Mitochondrion, 2013 ; 13(6):721-8.

5. Kiuru-Enari, S., \& Haltia, M. Hereditary gelsolin amyloidosis. Peripheral Nerve Disorders (1st ed., 2013 ; Vol. 115, pp. 659-681). Elsevier B.V

6. Foley, A. R., Menezes, M. P., Pandraud, A., Gonzalez, M. A., Al-Odaib, A., Abrams, A. J., et al. (2013). Treatable childhood neuronopathy caused by mutations in riboflavin transporter RFVT2. Brain 2013 ; 137(1) : 44-56.

7. Sathasivam, S. (2008). Brown-Vialetto-Van Laere syndrome. Orphanet Journal of Rare Diseases. https://doi.org/10.1186/1750-1172-3-9.

8. Bosch, A. M., Stroek, K., Abeling, N. G., Waterham, H. R., ljlst, L., \& Wanders, R. J. A. (2012). The Brown-Vialetto-Van Laere and Fazio Londe syndrome revisited: Natural history, genetics, treatment and future perspectives. Orphanet Journal of Rare Diseases. Orphanet Journal of Rare Diseases. https://doi.org/10.1186/1750-1172-7-83.

9. Johnson, J. O., Gibbs, J. R., Megarbane, A., Urtizberea, J. A., Hernandez, D. G., Foley et al. Exome sequencing reveals riboflavin transporter mutations as a cause of motor neuron disease. Brain, 2012 ; 135(9) : 2875-2882. https://doi.org/10.1093/brain/aws161.

10. Bosch, A. M., Abeling, N. G. G. M., ljlst, L., \& Knoester, H. (2011). Brown-Vialetto-Van Laere and Fazio Londe syndrome is associated with a riboflavin transporter defect mimicking mild MADD : a new inborn error of metabolism with potential treatment, 159-164. https://doi.org/10.1007/s10545010-9242-z.

11. Barile, M., Giancaspero, T. A., Leone, P., Galluccio, M., Indiveri, C. Riboflavin transport and metabolism in humans. Journal of Inherited Metabolic Disease 2016; 39(4): 545-557.

https://doi.org/10.1007/s10545-016-9950-0.

12. Olsen, R. K. J., Olpin, S. E., Andresen, B. S., Miedzybrodzka, Z. H., Pourfarzam, M., Frerman, F. E., et al. (2007). ETFDH mutations as a major cause of riboflavin-responsive multiple acyl-CoA dehydrogenation deficiency. Brain 2007; 130: 2045-2054. https://doi.org/10.1093/brain/awm135. 


\section{Légendes.}

Vidéo 1 : Amyotrophie linguale et très discrets mouvements anormaux péribuccaux.

Vidéo 2 : Mouvements anormaux péribuccaux.

Figure 1. Représentation simplifiée du métabolisme de la riboflavine. RF : riboflavine; RFVT : transporteur de la riboflavine (1,2,3); FAD: Flavine Adénine Dinucléotide; FMN : Flavine MonoNucléotide. 

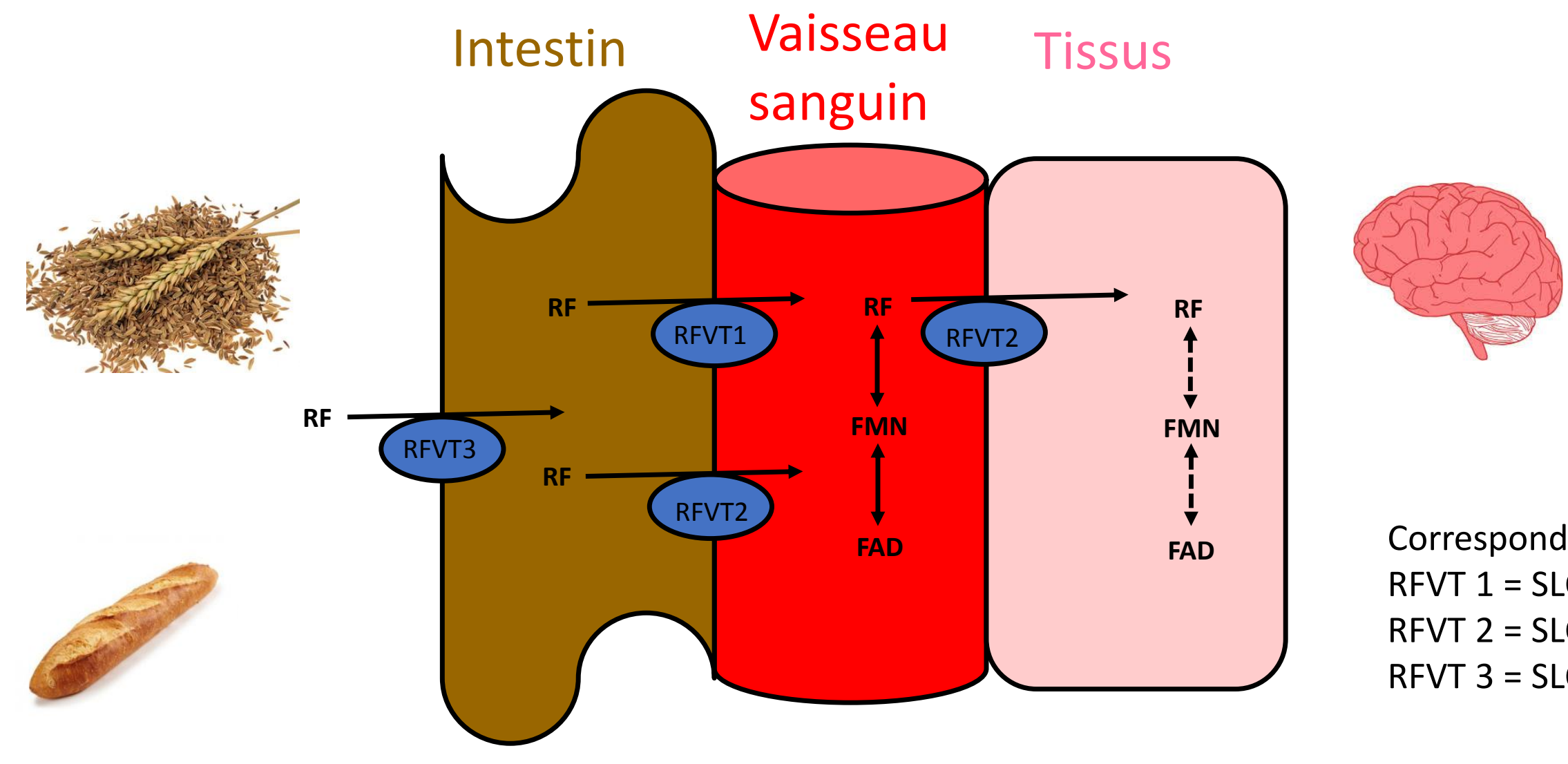

Correspondance génétique RFVT 1 = SLC52A1 RFVT $2=$ SLC52A2 RFVT $3=$ SLC52A3 